\title{
Laparoscopic management of adnexal torsion in a twin, in vitro fertilization pregnancy at 23 weeks
}

\author{
Turgut Aydin, Burak Yucel \\ Assisted Reproduction Unit, Acibadem Hospital, Kayseri, Turkey
}

Videosurgery Miniinv 2014; 9 (4): 655-657

DOI: $10.5114 /$ wiitm.2014.45732

\begin{abstract}
Maternal adnexal torsion in pregnancy, with a reported incidence of 1-10 per 10,000 spontaneous pregnancies, is very uncommon. Since it has been described as a severe complication after controlled ovarian hyper-stimulation for in vitro fertilization (IVF), it is more common in IVF pregnancies. This condition mainly occurs in the first trimester; it is rare during the late second trimester. Conservative management of adnexal torsion by laparoscopy is extended for the management of such conditions during pregnancy. An enlarged uterus could cause operational difficulties in the second trimester. In this paper, we report a case of adnexal torsion in a twin, 23 weeks with IVF pregnancy, that was successfully untwisted by laparoscopy.
\end{abstract}

Key words: adnexal torsion, ovarian stimulation, pregnancy, gynecological laparoscopy.

\section{Introduction}

Adnexal torsion (AT) is an uncommon cause of surgical emergency during pregnancy. Its incidence is one in 5000 pregnancies. The incidence is highest during the first trimester of pregnancy, the condition being rare during the second and third trimester [1]. After ovulation induction, the risk of AT is assumed to be increased. AT presenting as acute abdomen in pregnancy must be surgically managed [2].

In this paper, we report a case of adnexal torsion in a twin, 23 weeks, in vitro fertilization (IVF) pregnancy, that was successfully untwisted by laparoscopy.

\section{Case report}

A 28-year-old woman with a history of primary infertility for 4 years was referred to our IVF Center after three unsuccessful attempts. The couple was included in an IVF cycle. The indication was male infertility and she has polycystic ovaries. Controlled ovarian hyperstimulation was performed using a GnRH antagonist protocol with mild stimulation that involved the administration of recombinant FSH, 150 IU daily. The stimulation protocol permitted the retrieval of 16 oocytes after ovulation triggering with human choriogonadotropin $\alpha$. Two embryos were transferred. She conceived twins and her pregnancy until 23 weeks was uneventful.

At 23 weeks of gestation, she presented with right flank and lower abdominal pain of $12 \mathrm{~h}$ duration and had nausea and vomiting after the onset of pain. There was no vaginal bleeding, uterine contractility, or any bowel symptoms. She was afebrile $\left(36.4^{\circ} \mathrm{C}\right)$. Physical examination showed a point of maximal tenderness in the right lower abdominal quadrant. Deep palpation on that side provoked abdominal guarding. Bimanual examination showed a gravid uterus of 25 weeks size and a normal cervical status. The laboratory workup showed a count of 18000 white blood cells per microliter

\section{Address for correspondence}

Burak Yucel MD, Assisted Reproduction Unit, Acibadem Hospital, Seyit Gazi M., MKP Bulvari, No: 1, 38100 Kayseri, Turkey,

phone: +90 5326009554, e-mail: drburakyucel@gmail.com 
whereas hepatic enzymes, hematocrit and urine analysis were normal. Ultrasonography showed live twin fetuses at 23 weeks of gestation, with a right sided adnexal mass of $5-6 \mathrm{~cm}$ with mixed echogenicity and free fluid in the pouch of Douglas. Doppler mapping showed absent right ovarian vascular flow. Acute appendicitis and renal colic were excluded.

The patient was taken up for emergency laparoscopy with a presumptive diagnosis of right adnexal torsion. Pneumoperitoneum was created using a Veress needle at Palmer's point to prevent injury to the gravid uterus. A central port of $5 \mathrm{~mm}$ was created in the midline $3-4 \mathrm{~cm}$ above the umbilicus with one secondary port of $5 \mathrm{~mm}$ each on the left side at the level of the umbilicus. Lateral Trendelenburg positions and changing the probe of the camera were used to improve visualization. The laparoscopic findings showed the right adnexa twisted three times at the pedicle. The enlarged cystic ovary had a bluish hue. The surgeons proceeded to unwind the adnexa, pushing the ovary in the opposite direction of the torsion. After a few minutes, there was re-coloration
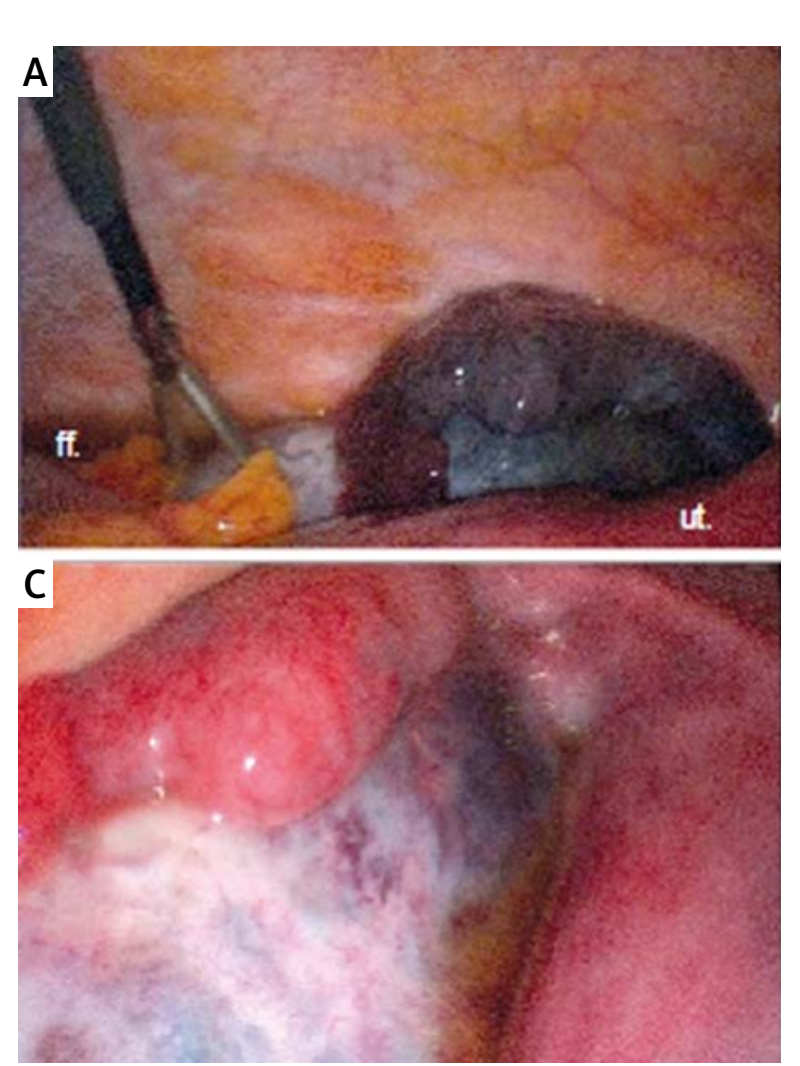

and a decrease of the adnexal edema, signs of a successful recovery, and the ovary was preserved.

The operation was completed within $10 \mathrm{~min}$. No tocolysis was needed postoperatively. She was discharged home in $12 \mathrm{~h}$. The remaining course of pregnancy was normal and she delivered two healthy children of $2550 \mathrm{~g}$ and $2600 \mathrm{~g}$ at 35 weeks of gestation. In cesarean section the right ovary was totally normal. The postpartum was also uneventful (Photo 1).

\section{Discussion}

The AT mainly occurs during the first trimester of pregnancy. However, ovarian masses that persist into the second trimester have more risk for torsion [3]. Some investigators have suggested that the correct diagnosis of AT might be delayed in the second or third trimester because the increased dimensions of the uterus cause difficulties in abdominal palpation and inefficient ultrasonographic examination.

Some studies have reported that ovulation induction increases the risk for maternal AT in pregnancy. After ovarian stimulation, the incidence of AT in
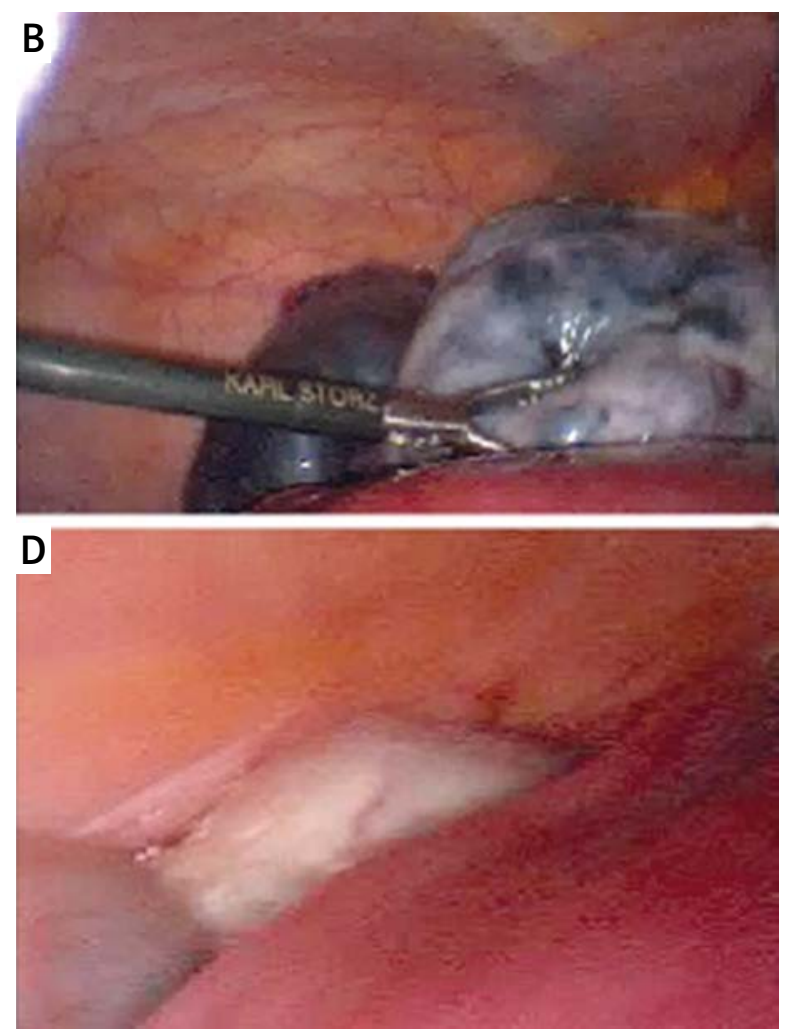

Photo 1. A - Torsioned right ovary, B - detorsion of ovary, C - 3 min after detorsion, D - normal left ovary

ut. - uterus, ff. - free fluid 
pregnancy rises to $6 \%$, reaching up to $16 \%$ in cases of ovarian hyperstimulation syndrome $[4,5]$. Our patient had received ovulation induction prior to pregnancy and there was an excessive response (16 oocytes retrieved), but she was not complicated by ovarian hyperstimulation. Increased risk can be due to the bilaterally enlarged ovaries with multiple follicular or lutein cysts in patients, especially in those who become pregnant with persistent cysts.

Ultrasound scanning is usually the first imaging tool used in evaluating pelvic pain during pregnancy. An enlarged twisted ovary could be identified by ultrasound in almost all patients (95\%) [6]. Doppler flow imaging has been shown to improve the accuracy of diagnosing AT outside pregnancy, while during pregnancy this imaging technique had higher false-negative rates [3].

Laparoscopy is a safe and effective surgical approach to treating AT in both pregnant and non-pregnant women. Laparoscopy did not have an adverse impact on maternal and fetal outcomes [7]. It is clear that compared to laparotomy, laparoscopy may be better tolerated by the patient due to minimal postoperative discomfort and the avoidance of a scar in the presence of a growing abdomen [8].

Laparoscopy in the second trimester can have some difficulties: possible injury to the enlarged uterus, or cardiovascular and respiratory alterations during the pneumoperitoneum. Penetrative injuries are likely to occur at the beginning of the procedure with the Veress needle, and subsequently the trocar. During advanced pregnancy, the best choice may be insertion of the needle from the palmar point or the performance of an open laparoscopy [3]. We chose the first option in our case.

\section{Conclusions}

Ovulation induction is a risk factor for adnexal torsion in IVF pregnancies. A high index of suspicion and awareness throughout pregnancy should be recommended, particularly if the patient experiences lower abdominal pain and ultrasonographic findings are present. Laparoscopy for AT, performed by trained surgeons, is safe and effective in the second trimester.

\section{References}

1. Ramesh B, Geeta U, Kavitha C. Successful laparoscopic management of adnexal torsion during pregnancy: a case report. J Obs Gynecol India 2010; 60: 337-8.
2. Hoover K, Jenkins TR. Evaluation and management of adnexal mass in pregnancy. Am J Obstet Gynecol 2011; 205: 97-102.

3. Hasson J, Tsafrir Z, Azem F, et al. Comparison of adnexal torsion between pregnant and nonpregnant women. Am J Obstet Gynecol 2010; 202: 536.e1-6.

4. Bider D, Mashiach S, Dulitzky M, et al. Clinical, surgical and pathologic findings of adnexal torsion in pregnant and nonpregnant women. Surg Gynecol Obstet 1991; 173: 363-6.

5. Mashiach S, Bider D, Moran O, et al. Adnexal torsion of hyperstimulated ovaries in pregnancies after gonadotropin therapy. Fertil Steril 1990; 53: 76-80.

6. Chang SD, Yen CF, Lo LM, et al. Surgical intervention for maternal ovarian torsion in pregnancy. Taiwan J Obstet Gynecol 2011; 50: 458-62.

7. Smorgick N, Pansky M, Feingold M, et al. The clinical characteristics and sonographic findings of maternal ovarian torsion in pregnancy. Fertil Steril 2009; 92: 1983-7.

8. Bassil S, Steinhart U, Donnez J. Successful laparoscopic management of adnexal torsion during week 25 of a twin pregnancy. Hum Reprod 1999; 14: 855-7.

Received: 1.08.2014, accepted: 20.08.2014. 